\title{
Compensating the effects of FES-induced muscle fatigue by rehabilitation robotics during arm weight support
}

DOI 10.1515/cdbme-2017-0007

\begin{abstract}
Motor functions can be hindered in consequence to a stroke or a spinal cord injury. This often results in partial paralyses of the upper limb. The effectiveness of rehabilitation therapy can be improved by the use of rehabilitation robotics and Functional Electrical Stimulation (FES). We consider a hybrid arm weight support combining both. In order to compensate the effect of FES-induced muscle fatigue, we introduce a method to substitute the decreasing level of FES support by cable-driven robotics. We evaluated the approach in a trial with one healthy subject performing repetitive arm lifting. The controller automatically adapted the support and thus no increase in user generated volitional effort was observed when FES induced muscle fatigue occured.
\end{abstract}

Keywords: upper limb rehabilitation; functional electrical stimulation; rehabilitation robotics; muscle fatigue.

\section{Introduction}

A stroke or a spinal cord injury (SCI) can result in injuries of the central nervous system (CNS), which may lead to a partial or complete loss of motor function in parts of the body. Rehabilitation therapy has been shown to improve recovery of motor functions after a stroke or SCI $[1,2]$ Two already widely used methods during rehabilitation therapy are Functional Electrical Stimulation (FES) and rehabilitation robotics. Robotic systems are mostly used to provide external assistance to the paralyzed limb in order to facilitate movement. FES also supports the patient during motion, with the advantage that it activates the muscles,

\footnotetext{
*Corresponding author: Arne Passon, Control Systems Group, Technische Universität Berlin, Einsteinufer 17, EN11, Berlin, Germany, E-mail: passon@control.tu-berlin.de

Paul Meyer-Rachner, Christian Klauer and Thomas Schauer: Control Systems Group, Technische Universität Berlin, Einsteinufer 17, EN11, Berlin, Germany
}

thereby strengthening them while retarding muscle atrophy and reducing spasticity [3]. It also improves proprioception and kinesthetic feedback. However, the trajectory of an FES assisted movement is harder to control [4]. Another main disadvantage of using FES during therapy is the quick occurrence of muscle fatigue since the CNS activates muscle fibers in a much more efficient way than FES does [5].

Studies have proven the effectiveness of both methods in improving the recovery of motor functions $[6,7]$. In addition, recent research shows that combining robotic assisted rehabilitation with FES further increases the benefits of rehabilitation therapy $[5,8]$.

Our approach uses the support provided by the robotic system to compensate the effects of FES-induced muscle fatigue. During longer therapy sessions, the stimulation intensity required to achieve a certain level of muscle activity increases as the electrically stimulated muscles get fatigued. In this case, we increase the proportion of support provided by the robotics.

\section{Methods}

\subsection{Experimental setup}

Experiments were performed using the setup shown in Figure 1. A stimulator (RehaStim, Hasomed GmbH, Germany), an active cable-driven robotic system (Diego, Tyromotion $\mathrm{GmbH}$, Austria), an EMG Amplifier (Porti 32, TMSi, Netherlands) and a computer running Linux were used. The control algorithms were implemented in Simulink ${ }^{\circledR}$ (Mathworks, USA) and a modified Linux real-time target was used to generate an executable binary that runs in soft real-time at $25 \mathrm{~Hz}$. Internal variables and parameters were visualized and set using a Python-GUI.

The stimulation pulses were transmitted to the muscle via two self-adhesive hydro-gel transcutaneous electrodes overlapping the anterior and medial part of the deltoid muscle. The stimulator generated bi-phasic stimulation pulses of current $I \in[0,100] \mathrm{mA}$ and pulse width $p w \in[100$, 

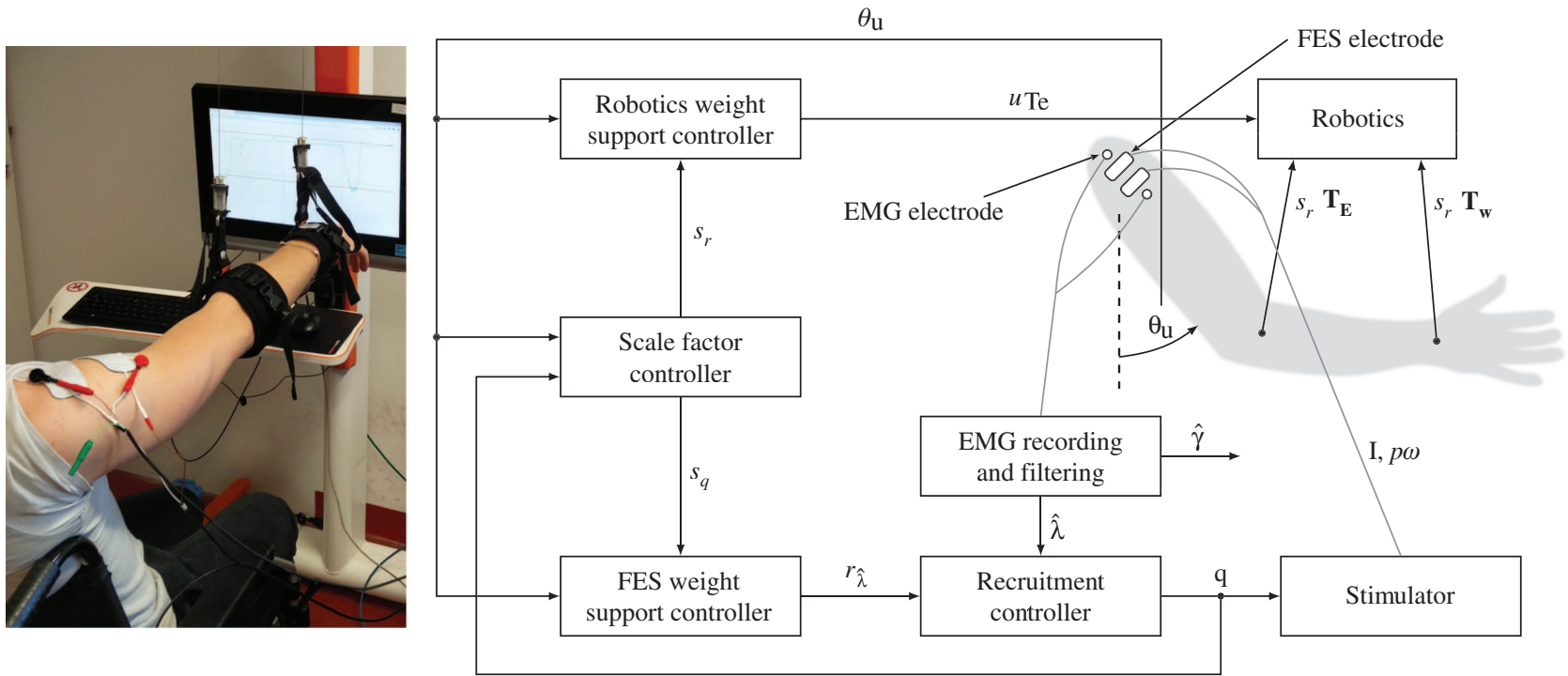

Figure 1: Experimental Setup: The required elbow rope tension $u_{\mathrm{Te}}$ and the recruitment level $r_{\hat{\lambda}}$ to hold the arm at a shoulder flexion angle $\theta_{\mathrm{u}}$ are scaled with the support factors $s_{r}$ and $s_{q}$ respectively. The total scale factor $s_{\text {total }}=s_{r}+s_{q}$ has to be within [0, 1]. The EMG is recorded and processed to obtain the FES induced muscle recruitment level $\hat{\lambda}$ and the volitional muscle activity $\hat{\gamma}$. The recruitment controller calculates the required stimulation intensity $q$ which is then generated by the stimulator using the current $I$ and the pulse width $p w$. The cable-driven robotics generates the tension vectors $s_{r} T_{E}$ and $s_{r} T_{W}$.

500] $\mu$ s. Thereby $I$ and $p w$ were computed to realize the normalized pulse charge $q$ according to [9].

Two ropes of the robotics system were attached to the forearm's elbow and wrist joints. The actuators, located above the user, apply a tension to the ropes and attached sensors provided data from which the position of the arm and thus the shoulder flexion angle $\theta_{\mathfrak{u}}$ were calculated.

\subsection{Control idea}

Two control algorithms provide adaptive weight support to the user's arm. The first control algorithm calculates the rope tensions required to hold the arm at the current angle $\theta_{\mathrm{u}}$. A second algorithm computes the needed stimulation intensity to maintain $\theta_{\mathrm{u}}$. Both methods are scaled by factors to determine their amount of weight support provided to the user's arm. A scale factor controller adapts these two factors if FES-induced muscle fatigue is detected.

\subsection{FES weight support controller}

The virtual weight compensation using FES is implemented as presented in [10]. The FES induced muscle recruitment level $\hat{\lambda}$ is estimated from the measured evoked EMG. During an identification routine, the required $\hat{\lambda}$ for each $\theta_{\mathfrak{u}} \in[40$, $100]^{\circ}$ is obtained. A discrete-time integrating controller with anti-windup strategy is chosen as recruitment controller. It adjusts the stimulation intensity $q$ depending on the current muscle activity $\hat{\lambda}$ and the required muscle activity $r_{\hat{\lambda}}\left(\theta_{\mathrm{u}}\right)$, whereby $q$ is limited by the maximum tolerated stimulation intensity $q_{\max }$ of the subject.

As muscle fatigue occurs, the muscle recruitment level $\hat{\lambda}$ generated by a certain value of $q$ decreases. To achieve a certain $\theta_{\mathrm{u}}$, the recruitment controller increases the stimulation intensity $q$ in order to obtain the muscle activity required to hold the arm at this angle. The patient dependent FES support factor $s_{q} \in[0,1]$ scales the provided support to an appropriate level.

\subsection{Robotics weight support controller}

The weight support applied by the robotics to compensate the gravitational forces of the arm is implemented as described in (11). The required rope tension at the elbow $\left\|\mathbf{T}_{\mathrm{E}}\right\|_{2}$ is calculated by a static arm model depending on $\theta_{\mathrm{u}}$, where $\|. .\|_{2}$ denotes the euclidean norm. From experience, the rope tension at the wrist $\left\|\mathbf{T}_{\mathrm{W}}\right\|_{2}$ is chosen as $0.55\left\|\mathbf{T}_{\mathrm{E}}\right\|_{2}$, which achieves an almost horizontal forearm posture.

In contrast to [11], no additional identification routine is necessary due to an alternative determination of the necessary arm parameters. The weights of the upper arm and forearm are considered to be a percentage of the total body weight [12]. The lengths of both arm segments are 
measured by means of the robotics at the beginning of each experiment. The patient dependent factor $s_{r} \in[0,1]$ scales the provided elbow rope tension to $u_{\mathrm{Te}}=s_{r}\left\|\mathbf{T}_{\mathrm{E}}\right\|_{2}$.

\subsection{Scale factor controller}

Our approach assumes that the user alternates between holding his arm at a lower angle and an upper angle by following a given reference for $\theta_{\mathrm{u}}$. The reference is shown to the subject, who is asked to follow it by using his residual voluntary activity. The scale factor controller updates the scale factors $s_{q}$ and $s_{r}$ at the end of every iteration $k$ after the subject's arm comes down from the upper angle. The mean of $q$ during the phase when the upper angle is reached, is averaged over the last three iterations to yield $\bar{q}_{\text {high }}[k]$. If it exceeds a patient dependent threshold $\sigma_{q}$, the FES scale factor is reduced and coincidentally the robotics scale factor is increased.

To achieve this, the FES scale factor $s_{q}$ is adapted by an integrating controller as

$$
s_{q}[k+1]=\operatorname{sat}_{0, s_{q, \text { init }}}\left(s_{q}[k]+K_{I} \cdot e[k]\right),
$$

where $K_{I}$ is the adjustable gain of the integrator and the error $e[k]$ is defined as

$$
e[k]=\sigma_{q}-\bar{q}_{\text {high }}[k] .
$$

Thereby $s_{q}$, init is the initial value of $s_{q}$ at the beginning of the experiment. The total support level $s_{\text {total }}=s_{r}+s_{q}$ is maintained by $s_{r}[k]=s_{\text {total }}-s_{q}[k]$.

\subsection{Experimental procedure}

The complete setup was implemented as described in Section 2.1. At first, the maximum tolerated stimulation intensity $q_{\max }$ of the subject was detected by increasing $q$ until the subject reported discomfort. Then $q$ was increased until $q_{\max }$ was reached and afterwards decreased to zero to identify the required $\hat{\lambda}$ for each $\theta_{\mathrm{u}} \in[40,100]^{\circ}$. For a patient, the therapist would now manually test with which setting of $s_{\text {total }}$ the patient would just be able to reach the desired upper angle while exerting the maximum of his volitional effort. Thereby, a higher $s_{q}$ than $s_{r}$ should be intended. $\sigma_{q}$ should be chosen as $90 \%$ of $q_{\max }$ to allow, if necessary, short excursions above $\sigma_{q}$. Nevertheless, this has to be evaluated in experiments with patients.

For the healthy subject, a lower threshold $\sigma_{q}=0.75 q_{\text {max }}$ and at the same time a high $s_{\text {total }}=0.9$ were chosen. Thus, it was possible to provoke relatively quickly occurring muscle fatigue. The subject had to complete 20 iterations of a movement, successively raising and lowering the arm to achieve a shoulder flexion angle $\theta_{\mathrm{u}}$ that is within two reference bands at $50^{\circ} \pm 5^{\circ}$ (lower angle) and $90^{\circ} \pm 5^{\circ}$ (upper angle). For this reason, the actual desired reference band was visualized to the subject.

\section{Results}

The results of the trial with one healthy subject (age 21 years) are shown in Figure 2. FES-induced muscle fatigue occurred starting at the iteration around 90-100 s, whereby the recruitment controller increased $q$ to achieve the scaled desired muscle recruitment level $s_{q} \hat{\lambda}$ belonging to the upper flexion angle. As a result, $\bar{q}_{\text {high }}$ exceeded the threshold $\sigma_{q}$ after this iteration. Consequently the FES scale factor $s_{q}$ started to be gradually reduced until $\bar{q}_{\text {high }}$ falls back below the threshold.

The estimated volitional muscle activity $\hat{\gamma}$, which is calculated as described in [13] allows an evaluation of the contributed effort by the subject himself. During the phase where the scale factors are adjusted, $\hat{\gamma}$ remains roughly the same. From this it can be concluded that the user did not have to provide more effort to perform the same movement and thus the decreased support of the FES is well compensated by the increased support of the robotics.

\section{Conclusion}

The use of a robotic system to compensate the effects of muscle fatigue appears to be a promising approach to eliminate one of the biggest drawbacks of FES during arm lifting. Further trials with stroke or SCI patients have to be performed in order to confirm the effectiveness of the procedure. Due to the fast setup and only one containing fast identification routine, the presented method is relatively simple to apply. The main limitation of the current approach is that changes in $q$ are only analyzed when the flexion angle is near the chosen upper angle. More work needs to be done on tracking muscle fatigue during arbitrary movements. A further development using the approach of [13] will enable longer and therefore more efficient therapy sessions.

Acknowledgment: We would like to thank Axelgaard Manufacturing Co., USA for donating the used stimulation electrodes. 

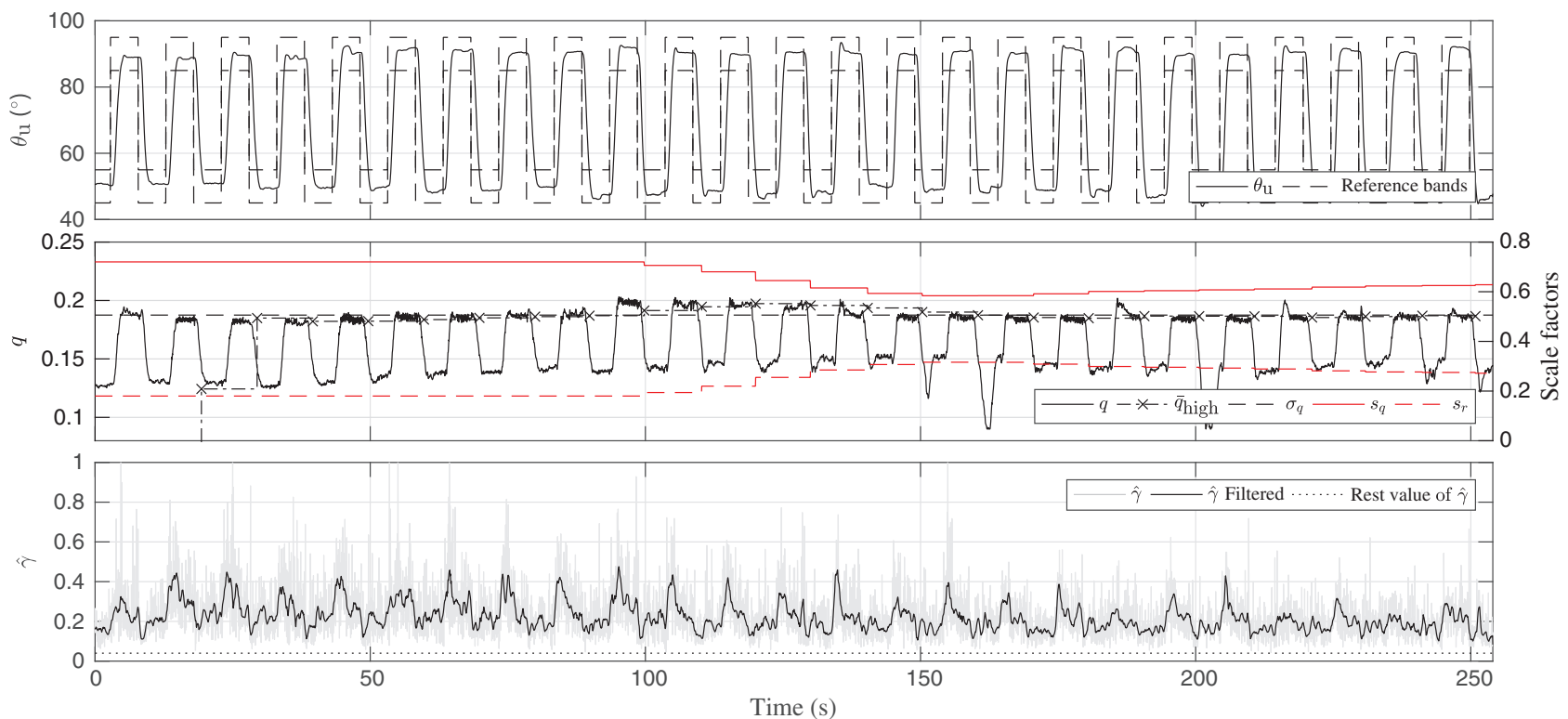

Figure 2: Experimental results of a trial with a healthy subject. The subject had to hold $\theta_{u}$ between two visualized reference bands. $\hat{\gamma}$ is an estimate of the volitional muscle activity based on the measured EMG and was filtered using a median and moving average filter. The rest value of $\hat{\gamma}$ corresponds to the value of $\hat{\gamma}$ when no volitional movement is made. The parameters for this measurement were $q_{\max }=0.25$, $K_{l}=1 / q_{\max }=4$. The scale factors were set to $s_{\text {total }}=0.9, s_{q, \text { init }}=0.72$ and $s_{r}$, init $=0.18$.

\section{Author's Statement}

Research funding: The work was conducted within the research project BeMobil, which is supported by the German Federal Ministry of Education and Research (BMBF) (FKZ16SV7069K). Conflict of interest: Authors state no conflict of interest. Informed consent has been obtained from all individuals included in this study. Ethical approval: The research related to human use complies with all the relevant national regulations, institutional policies and was performed in accordance with the tenets of the Helsinki Declaration. The performed trials have been approved by the ethics committee of the Berlin Chamber of Physicians.

\section{References}

[1] Feys HM, De Weerdt WJ, Selz BE, Cox Steck GA, Spichiger R, Vereeck LE, et al. Effect of a therapeutic intervention for the hemiplegic upper limb in the acute phase after stroke. Stroke 1998;29:785-92.

[2] Sumida M, Fujimoto M, Tokuhiro A, Tominaga T, Magara A, Uchida R. Early rehabilitation effect for traumatic spinal cord injury. Arch Phys Med Rehabil. 2001;82:391-5.

[3] Doucet BM, Lam A, Griffin L. Neuromuscular electrical stimulation for skeletal muscle function. Yale J Biol Med. 2012;85:201-15.

[4] Vette AH, Masani K, Kim JY, Popovic MR. Closed-loop control of functional electrical stimulation-assisted arm-free standing in individuals with spinal cord injury: a feasibility study. Neuromodulation 2009;12:22-32.
[5] Hu XL, Tong RKY, Ho NSK, Xue JJ, Rong W, Li LSW. Wrist rehabilitation assisted by an electromyography-driven neuromuscular electrical stimulation robot after stroke. Neurorehabilitation and Neural Repair 2015;29:767-76.

[6] Kawashima N, Popovic MR, Zivanovic V. Effect of intensive functional electrical stimulation therapy on upper-limb motor recovery after stroke: Case study of a patient with chronic stroke. Physiother Can 2013;65:20-8.

[7] Volpe BT, Lynch D, Rykman-Berland A, Ferraro M, Galgano M, Hogan $N$, et al. Intensive sensorimotor arm training mediated by therapist or robot improves hemiparesis in patients with chronic stroke. Neurorehabil Neural Repair. 2008;22:305-10.

[8] Miyasaka H, Orand A, Ohnishi H, Tanino G, Takeda K, Sonoda S. Ability of electrical stimulation therapy to improve the effectiveness of robotic training for paretic upper limbs in patients with stroke. Med Eng Phys. 2016;38:1172-5.

[9] Klauer C, Raisch J, Schauer T. Linearisation of electrically stimulated muscles by feedback control of the muscular recruitment measured by evoked emg. Proc. of the 17 th International Conference on Methods and Models in Automation and Robotics, IEEE, Międzyzdroje, Poland; 2012. p. 108-13.

[10] Klauer C, Passon A, Raisch J, Schauer T. Virtual weightcompensating exoskeleton using $\lambda$-controlled fes. Proc. of Automed 2013, Dresden, Germany; 2013.

[11] Knuth S, Passon A, Dähne F, Niedeggen A, Schmehl I, Schauer T. Adaptive arm weight support using a cable-driven robotic system. Springer International Publishing; 2017. p. 1317-21.

[12] de Leva P. Adjustments to zatsiorsky-seluyanov's segment inertia parameters. J Biomech. 1996;29:1223-30.

[13] Klauer C, Ferrante S, Ambrosini E, Shiri U, Dähne F, Schmehl I, et al. A patient-controlled functional electrical stimulation system for arm weight relief. Med Eng Phys. 2016;38:1232-43. 